\title{
Clinical Significance of Plasma Levels of Soluble CD40 in Patients with Chronic Lymphocytic Leukemia
}

\author{
Barry D. Hock ${ }^{1}$, Liam J. Fernyhough ${ }^{2}$, Sheryl M. Gough ${ }^{3 *}$, Judith L. McKenzie ${ }^{1}$ \\ ${ }^{1}$ Haematology Research Group, Christchurch Hospital, Christchurch, New Zealand; ${ }^{2}$ Pathology Department, University of Otago, \\ Christchurch, New Zealand; ${ }^{3}$ Cancer Genetics Research Group, University of Otago, Christchurch, New Zealand. \\ Email: barry.hock@otago.ac.nz
}

Received December $12^{\text {th }}, 2012$; revised January $16^{\text {th }}, 2013$; accepted February $1^{\text {st }}, 2013$

\begin{abstract}
CD40 ligation provides signals central to chronic lymphocytic leukemia (CLL) cell survival and proliferation. The release of soluble CD40 (sCD40) may impact on both these signals and CD40 targeted therapies. In this study we investigated the prognostic significance of plasma SCD40 in untreated CLL patients $(\mathrm{n}=34)$. We report that $56 \%$ had levels higher than those of normal donors and that elevated levels were associated with significantly shorter treatment free (TFS) and overall survival. In bivariate analysis SCD40 remained a significant marker of TFS independent of Binet staging, CD38 positivity and lymphocyte count. RT-PCR analysis demonstrated that CLL cells expressed transcripts encoding putative sCD40. These results suggest SCD40 may play a role in CLL progression and that its function, prognostic significance and potential impact on antibody based therapies should be further investigated.
\end{abstract}

Keywords: CD40; Chronic Lymphocytic Leukemia; Survival

\section{Introduction}

The trans-membrane molecule CD40 has attracted attention as a therapeutic target in CLL [1]. Within the haematopoietic system the CD40-CD40 ligand (CD40L) interaction plays a central role in immune regulation [2]. The majority of CLL cells strongly express membrane CD40 (mCD40), and mCD40-CD40L engagement within the lymph node microenvironment is thought to provide signals critical for their proliferation and survival [1]. Ligation of mCD40 on CLL cells has been reported to markedly modify their phenotype, function, proliferation, apoptosis and sensitivity to chemotherapy/antibody therapy. These effects have stimulated the clinical investigation of novel CD40 therapies such as CD40 antibodies and CD40L transduced CLL cells $[1,3,4]$. These studies have yielded promising results, but it is clear that a better understanding of the complex regulation of CD40 signalling is required.

The release of soluble forms of membrane molecules which can enhance or inhibit the signaling of their membrane bound counterpart provides a potentially powerful immunoregulatory mechanism. Soluble forms of CD40 (sCD40) can potentially be generated by either proteolytic cleavage or alternative splicing although little is known of the expression of sCD40 transcripts by

${ }^{*}$ Current address: Genetics Branch, NCI/NIH, Bethesda, USA. normal leucocyte subsets or CLL populations [5-8]. Circulating sCD40 has been detected in human plasma [9-12] and elevated circulating levels have been associated with decreased immune responses [10]. Studies using B cell derived and recombinant forms of SCD40 indicate that it can block mCD40-CD40L interaction and is a potent inhibitor of both in vivo and in vitro immune responses $[2,7]$. We have previously established that the plasma levels of sCD40 are elevated in a proportion of CLL patients and that activated CLL cells release SCD40 [12]. The functional impact of sCD40 in CLL is unknown but may potentially modulate CLL directed immunity and/or CLL survival. In addition because sCD40 provides an alternative, non-cellular, target for CD40 directed therapeutics it may reduce the effectiveness of CD40 specific therapies, particularly if sCD40 levels are higher in patients with more aggressive disease.

In order to better understand the potential role of sCD40 in CLL we have analysed both the prognostic significance of sCD40 in a group of previously untreated CLL patients and the expression of SCD40 transcripts by CLL cells.

\section{Methods}

Blood was collected from donors with appropriate informed consent according to Upper South B Ethical 
Committee (NZ) guidelines, into EDTA tubes and the plasma fraction recovered by centrifugation $(400 \mathrm{xg}, 5$ $\mathrm{min})$. Following further centrifugation $(10,000 \mathrm{~g}, 30 \mathrm{~min})$ non sedimented material was stored at $-70^{\circ} \mathrm{C}$ prior to analysis. Normal blood was obtained from healthy volunteer donors (age $16-60,52 \%$ male) to the New Zealand Blood Transfusion Service and had undergone comprehensive disease/infection screening. CLL patient blood was obtained from previously untreated patients attending the Christchurch Hospital Haematology Department. Diagnosis was based on standard morphological criteria and all patients were positive for CD5, CD19 and CD23. Patient clinical notes were reviewed for the date of first treatment for CLL. Therapies included chlorambucil, fludarabine, cyclophosphamide and CHOP therapy (cyclophosphamide, doxorubicin, vincristine and prednisone).

Human peripheral blood mononuclear cells (PBMC) were isolated from blood (EDTA) by centrifugation over Ficoll/Paque (Amersham Pharmacia Biotech, Uppsala, Sweden). T cells, NK cells, B cells and monocytes were prepared from normal donor (ND) PBMC by imunomagnetic selection as previously described [13] and dendritic cells generated from PBMC using GM-CSF + IL-4 + LPS [14]. PBMC from CLL patients were $>95 \%$ $\mathrm{CD} 19+, \mathrm{CD} 5+$ and were not purified further.

sCD40 levels in stored plasma were measured by sandwich ELISA and membrane CD40 by flow cytometry as previously described [12].

Total RNA was extracted (RNeasy, Qiagen), cDNA generated (SuperScript ${ }^{\circledR}$ III First-Strand Synthesis System, Invitrogen) and RT-PCR performed as described previously [13]. The primers used for amplifying all CD40 transcripts present were

5'-TGGTCCTGCCGCCTGGTCTCACC-3' and

5'-ATCCTCCTGGGTGACCGGTTGGC-3'. Transcripts encoding soluble CD40 were amplified using the primer 5'-TGCATGCA GAGAAAAACAGTACC-3' and a primer spanning the exon $5 / 7$ splice boundary 5'GATCCTGGGGACCTTGTCCAAGGG-3'. Cycling conditions were $94^{\circ} \mathrm{C}$ for 4 minutes, followed by 35 cycles of $94^{\circ} \mathrm{C}$ for 1 minute, $60^{\circ} \mathrm{C}$ for 30 seconds, $72^{\circ} \mathrm{C}$ for 1 minute per kilobase of template target with a final $72^{\circ} \mathrm{C}$ extension for 7 minutes.

\section{Results}

\subsection{Levels of sCD40 in CLL Patients}

All normal donor (ND) and patient plasma samples contained detectable sCD40 (Figure 1(a)). Levels in patients $(\mathrm{n}=34$, mean $\pm \mathrm{SD}=1200 \pm 484 \mathrm{pg} / \mathrm{ml}$, range $=$ $560-2791 \mathrm{pg} / \mathrm{ml})$ were significantly higher $(\mathrm{P}<0.0001)$ than those of ND $(\mathrm{n}=44$, mean $\pm \mathrm{SD}=719 \pm 121 \mathrm{pg} / \mathrm{ml}$, range $=536-1014 \mathrm{pg} / \mathrm{ml}$ ).

\subsection{Association of sCD40 Levels with Clinical Features}

sCD40 levels were not significantly correlated with either age or platelet levels but were strongly correlated with absolute lymphocyte count (ALC) and moderately correlated with hemoglobin levels (Table 1).

The association of sCD40 with clinical characteristics was analysed further following sub-division of patients based on their sCD40 levels. The cut-off level was set at the upper value detected in ND with patients below this

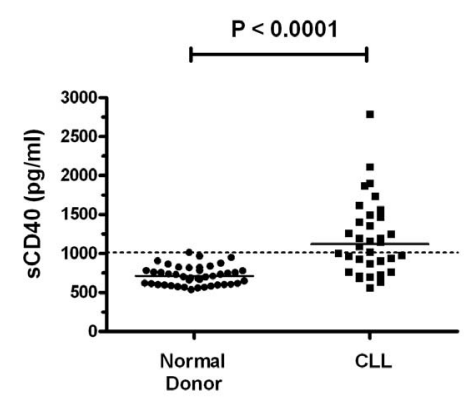

(a)

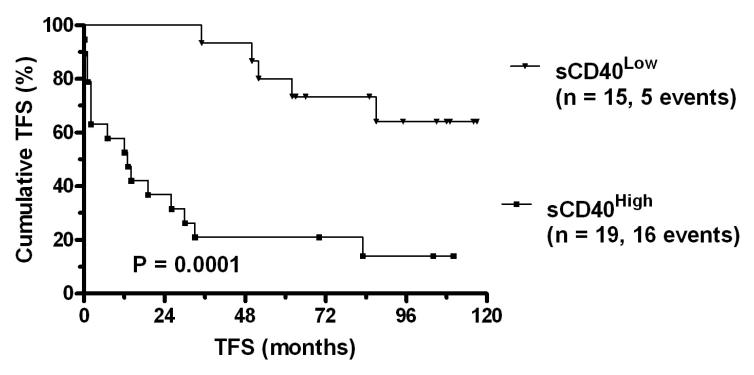

(b)

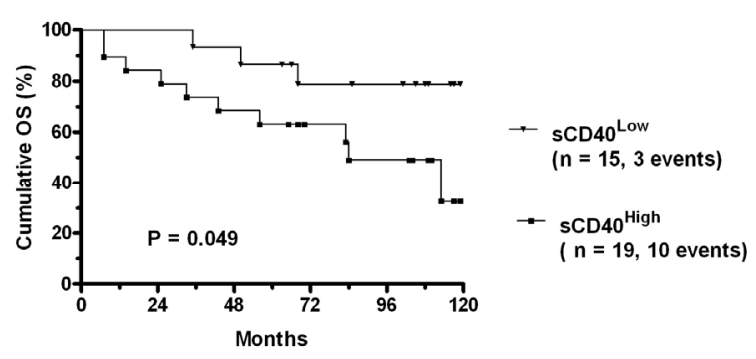

(c)

Figure 1. Treatment free and overall survival of CLL patients stratified according to SCD40 levels. (a) Scatter plot of sCD40 levels in the plasma of normal donors and CLL patients. The solid line indicates the median value in normal donors $(1710 \mathrm{pg} / \mathrm{ml})$ and patients $(1122 \mathrm{pg} / \mathrm{ml})$ and significance of difference in levels (Mann Whitney) indicated. The dashed line denotes the upper level observed in normal donors which was used as a cut-point to subdivide CLL patients into $\mathrm{SCD}^{\mathrm{High}}$ and $\mathrm{SCD}^{\mathrm{H}}{ }^{\mathrm{Low}}$ groups. The (b) treatment free survival and (c) overall survival of the $\mathrm{SCD} 40^{\mathrm{High}}$ and $\mathrm{SCD}^{4} 0^{\mathrm{Low}}$ groups are shown as Kaplan-Meier survival curves and the significance of overall survival differences is indicated. 
Table 1. Association of sCD40 levels with clinical hematological features in CLL patients.

\begin{tabular}{ccccc}
\hline Characteristic & \multicolumn{2}{c}{$\begin{array}{c}\text { a Correlation } \\
\text { with sCD40 }\end{array}$} & \multicolumn{2}{c}{$\begin{array}{c}\text { \% of patients } \\
\text { with characteristic }\end{array}$} \\
\hline R & P & All & $\begin{array}{c}\mathrm{sCD}^{\text {Low }} \text { vs } \\
\text { sCD40 }\end{array}$ \\
\hline Age & 0.22 & 0.203 & \\
ALC & $\mathbf{0 . 7 6}$ & $<\mathbf{0 . 0 0 1}$ & \\
Platelet & -0.25 & 0.159 & \\
Hemoglobin & $-\mathbf{0 . 4 8}$ & $\mathbf{0 . 0 0 5}$ &
\end{tabular}

\begin{tabular}{ccc} 
Age $\geq 65$ & $59 \%$ & $60 \%$ vs $48 \%$ \\
Sex $=$ male & $71 \%$ & $67 \%$ vs $74 \%$ \\
Lymphocytes $\geq 30$ & $32 \%$ & ${ }^{\text {b } 7 \% \text { vs } 53 \%}{ }^{* *}$ \\
CD $38+$ & $36 \%$ & $27 \%$ vs $44 \%$ \\
Binet Stage A & $82 \%$ & $93 \%$ vs $72 \%$ \\
Binet Stage C & $6 \%$ & $7 \%$ vs $5 \%$ \\
\hline
\end{tabular}

${ }^{\mathrm{a}}$ Spearman correlation; ${ }^{\mathrm{b}}$ The significance of differences between $\mathrm{SCD} 40^{\mathrm{Low}}$ and $\mathrm{SCD} 40^{\mathrm{High}}$ patient groups was determined by Chi square statistic. Bold indicates significant difference $(\mathrm{P}<0.05) ;{ }^{* *}$ indicates $\mathrm{P}<0.01$.

level defined as $\mathrm{sCD} 40^{\text {Low }}$ and those above as $\mathrm{SCD} 40^{\mathrm{High}}$.

There was no significant difference between $\mathrm{SCD} 40^{\text {Low }}$ and $\mathrm{SCD} 40^{\text {High }}$ groups with respect to the proportion with male sex, older age, Binet stage $\mathrm{C}$ or $\mathrm{CD} 38$ positivity (Table 1). However a significantly higher proportion of sCD $40{ }^{\text {High }}$ patients had elevated ALC.

\subsection{Association of sCD40 Levels with Treatment Free and Overall Survival}

In univariate analysis, $\mathrm{sCD} 40^{\mathrm{High}}$ patients had significantly shorter Treatment Free Survival (TFS) than sCD40 ${ }^{\text {Low }}$ group (Figure 1(b)). Other variables which were significant predictors of poor TFS were ALC $\geq 30$ $(\mathrm{P}=0.001)$, expression of $\mathrm{CD} 38(\mathrm{P}=0.006)$ and age $\geq$ $65(\mathrm{P}=0.031)$. Binet stage A was a significant marker of good prognosis $(\mathrm{P}<0.001)$.

The number of TFS events $(\mathrm{n}=21)$ precluded a full multivariate analysis. Bivariate analyses were therefore performed using $\mathrm{SCD} 40$ in combination with each of the other identified prognostic markers (Table 2). sCD40 remained a significant independent marker of TFS when included with CD38 status, Binet stage A or age $\geq 65$.

When included in an analysis with ALC $\geq 30, \mathrm{sCD} 40$ but not ALC $\geq 30$ remained a significant marker.

In univariate analysis of Overall Survival (OS) sCD40 ${ }^{\text {High }}$ patients had significantly shorter survival than ${ }_{\mathrm{sCD}} 40^{\mathrm{Low}}$ patients (Figure 1(c)). Other significant predictors of poor OS were CD38 expression $(\mathrm{P}=0.006)$ and age $\geq 65(\mathrm{P}=0.031)$, whilst staging as Binet stage $\mathrm{A}$ was a significant marker of good prognosis $(\mathrm{P}<0.001)$.
ALC $\geq 30$ was not a significant marker of OS. The number of OS events $(n=13)$ precluded bivariate analysis.

\subsection{Expression of Alternate CD40 Transcripts}

It has been reported that B cell lines express four CD40 transcripts, one of which lacks the transmembrane region and encodes a putative soluble form [6]. RT-PCR with primers spanning all CD40 exons detected strong expression of the full length transcript in all normal and CLL cell populations but did not clearly discriminate between smaller transcripts (Figure 2(a)). However a second RTPCR, utilising a primer specific for the sequence generated following deletion of the transmembrane region, clearly detected the sCD40 transcript in B cells, monocytes, DC and all three CLL populations analysed (Figure 2(b)). All of the CLL samples had strong expression of membrane CD40 and 2/3 were from patients with elevated $\mathrm{SCD} 40$ levels (data not shown).

Table 2. Bivariate analysis of treatment free survival in CLL patients.

\begin{tabular}{lccc}
\hline Analysis & Variables & HR $^{\mathbf{a}}$ & P value \\
\hline \multirow{2}{*}{ Model 1 } & CD38+ & $\mathbf{3 . 8}$ & 0.007 \\
& sCD40 & $\mathbf{8 . 7}$ & $<0.001$ \\
\multirow{2}{*}{ Model 2 } & Lymphocytes $\geq 30$ & 2.4 & 0.070 \\
& sCD40 & $\mathbf{4 . 7}$ & 0.005 \\
Model 3 & Binet Stage A & $\mathbf{0 . 1}$ & 0.001 \\
& sCD40 & $\mathbf{6 . 2}$ & 0.001 \\
Model 4 & Age $>65$ & $\mathbf{4 . 2}$ & 0.007 \\
& sCD40 & $\mathbf{8 . 0}$ & $<0.001$ \\
\hline
\end{tabular}

${ }^{\mathrm{a}}$ Hazard Ratio; ${ }^{\mathrm{a}}$ Bold indicates significant hazard ratio value $(\mathrm{P}<0.05)$.

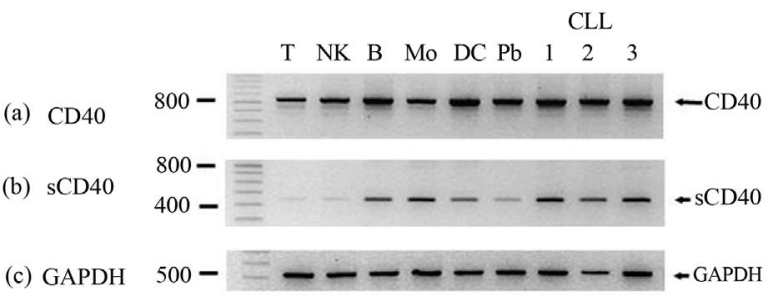

Figure 2. RT-PCR analysis of alternatively spliced CD40 transcripts. Semi quantitative RT-PCR was performed on purified normal cell populations $(T=T$ lymphocytes, $N K=$ natural killer cells, $B=B$ lymphocytes, $M o=$ monocytes, $\mathrm{DC}=$ dendritic cells, $\mathrm{Pb}=$ peripheral blood) and CLL cells from 3 patients using primers that amplify either (a) all CD40 transcripts; (b) sCD40 transcript; (c) GAPDH. Amplified products were visualised by ethidium bromide staining. A molecular weight ladder is shown on the left side and the position of bands and base pair indicated. Template negative (H20) controls for each PCR were run on the same gel but not shown. 


\section{Discussion}

In the current study we demonstrate that elevated levels of SCD40 were a significant marker of shorter TFS and OS in untreated CLL patients.

Current data suggest that the presence of sCD40 would inhibit immune responses [2]. The findings of this study, together with the association between high sCD40 levels and poor survival previously reported in AML and myeloma, provide further evidence to support this hypothesis [12]. However the possibility that SCD40 is not functionally relevant but is merely a marker of more aggressive or chemotherapy resistant malignancies cannot be discounted. Irrespective of its functional role, the results of the bivariate prognostic analysis reported in this study suggest that SCD40 merits further investigation as a clinically useful prognostic marker. In addition, the presence of SCD40 may potentially reduce the therapeutic effects of CD40 ligating reagents by providing an alternative pool of non-cellular targets and thereby reducing the interaction of the ligating reagents with membrane CD40. This type of interaction with the soluble forms of membrane molecules is thought to reduce the efficacy of therapeutic antibodies such as Rituximab and Campath-1H $[15,16]$. The possibility that elevated sCD40 levels may also contribute to the progression of other diseases in these patients must also be considered [17], particularly in view of the high frequency of comorbidities in CLL patients [18].

The finding that CLL cells express a transcript encoding a putative SCD40 indicates that CLL cells can potentially generate sCD40 from both alternate splicing and proteolytic cleavage of membrane CD40.

The observation, in this study, that the highest levels of sCD40 are present in patients with the poorest outcomes together with our previous finding that CD40 ligation can trigger $\mathrm{SCD} 40$ release by CLL cells suggests that the impact of sCD40 on CD40 directed therapies merits further investigation.

\section{Acknowledgements}

We wish to thank the volunteers and patients who donated blood samples. We acknowledge the contribution of clinical and laboratory staff of the Haematology Department, Christchurch Hospital in collecting patient samples and information. This study was supported by grants from the Maurice \& Phyllis Paykel Trust, Leukaemia \& Blood Cancer New Zealand and the Bone Marrow Cancer Research Trust.

\section{REFERENCES}

[1] R. E. Hayden, G. Pratt, C. Roberts, M. T. Drayson and C. M. Bunce, "Treatment of Chronic Lymphocytic Leuke- mia Requires Targeting of the Protective Lymph Node Environment with Novel Therapeutic Approaches," Leukemia \& Lymphoma, Vol. 53, No. 4, 2012, pp. 537-549. doi:10.3109/10428194.2011.610014

[2] A. Chatzigeorgiou, M. Lyberi, G. Chatzilymperis, A. Nezos and E. Kamper, "CD40/CD40L Signaling and Its Implication in Health and Disease," Biofactors, Vol. 35, No. 6, 2009, pp. 474-483. doi:10.1002/biof.62

[3] J. E. Castro, J. Melo-Cardenas, M. Urquiza, J. S. BarajasGamboa, R. S. Pakbaz and T. J. Kipps, "Gene Immunotherapy of Chronic Lymphocytic Leukemia: A Phase I Study of Intranodally Injected Adenovirus Expressing a Chimeric CD154 Molecule," Cancer Research, Vol. 72, 2012, pp. 2937-2948. doi:10.1158/0008-5472.CAN-11-3368

[4] J. C. Byrd, T. J. Kipps, I. W. Flinn, M. Cooper, O. Odenike, J. Bendiske, et al., "Phase I Study of the Anti-CD40 Humanized Monoclonal Antibody Lucatumumab (HCD122) in Relapsed Chronic Lymphocytic Leukemia," Leukemia \& Lymphoma, Vol. 53, No. 11, 2012, pp. 2136-2142.

[5] P. Esposito, T. Rampino, M. Gregorini, E. Gabanti, S. Bianzina and A. Dal Canton, "Mechanisms Underlying SCD40 Production in Hemodialysis Patients," Cellular Immunology, Vol. 278, No. 1-2, 2012, pp. 10-15. doi:10.1016/j.cellimm.2012.06.007

[6] M. Tone, Y. Tone, P. J. Fairchild, M. Wykes and H. Waldmann, "Regulation of CD40 Function by Its Isoforms Generated through Alternative Splicing," Proceedings of the National Academy of Sciences of the USA, Vol. 98, No. 4, 2001, pp. 1751-1756. doi:10.1073/pnas.98.4.1751

[7] D. Eshel, A. Toporik, T. Efrati, S. Nakav, A. Chen and A. Douvdevani, "Characterization of Natural Human Antagonistic Soluble CD40 Isoforms Produced through Alternative Splicing," Molecular Immunology, Vol. 46, No. 2, 2008, pp. 250-257. doi:10.1016/j.molimm.2008.08.280

[8] C. Contin, V. Pitard, T. Itai, S. Nagata, J. F. Moreau and J. Dechanet-Merville, "Membrane-Anchored CD40 Is Processed by the Tumor Necrosis Factor-Alpha-Converting Enzyme. Implications for CD40 Signaling," Journal of Biological Chemistry, Vol. 278, 2003, pp. 32801-32809. doi:10.1074/jbc.M209993200

[9] R. F. Schwabe, H. Engelmann, S. Hess and H. Fricke, "Soluble CD40 in the Serum of Healthy Donors, Patients with Chronic Renal Failure, Haemodialysis and Chronic Ambulatory Peritoneal Dialysis (CAPD) Patients," Clinical \& Experimental Immunology, Vol. 117, No. 1, 1999, pp. 153-158. doi:10.1046/j.1365-2249.1999.00935.x

[10] C. Contin, V. Pitard, Y. Delmas, N. Pelletier, T. Defrance, J. F. Moreau, et al., "Potential Role of Soluble CD40 in the Humoral Immune Response Impairment of Uraemic Patients," Immunology, Vol. 110, No. 1, 2003, pp. 131140. doi:10.1046/j.1365-2567.2003.01716.x

[11] Y. Zhuang, J. Huang, Z. Zhou, Y. Ge, Y. Fan, C. Qi, et al., "A Novel Blocking Monoclonal Antibody Recognizing a Distinct Epitope of Human CD40 Molecule," Tissue Antigens, Vol. 65, No. 1, 2005, pp. 81-87. doi:10.1111/j.1399-0039.2005.00342.x

[12] B. D. Hock, J. L. McKenzie, N. W. Patton, M. Drayson, K. Taylor, C. Wakeman, et al., "Circulating Levels and Clinical Significance of Soluble CD40 in Patients with 
Hematologic Malignancies," Cancer, Vol. 106, No. 10, 2006, pp. 2148-2157. doi:10.1002/cncr.21816

[13] B. D. Hock, L. J. Fernyhough, S. M. Gough, A. Steinkasserer, A. G. Cox and J. L. McKenzie, "Release and Clinical Significance of Soluble CD83 in Chronic Lymphocytic Leukemia," Leukemia Research, Vol. 33, No. 8, 2009, pp. 1089-1095. doi:10.1016/j.leukres.2009.01.001

[14] B. D. Hock, G. Roberts, J. L. McKenzie, P. Gokhale, N. Salm, A. D. McLellan, et al., "Exposure to the Electrofusion Process Can Increase the Immunogenicity of Human Cells," Cancer Immunology, Immunotherapy, Vol. 54, No. 9, 2005, pp. 880-890. doi:10.1007/s00262-004-0659-9

[15] T. Manshouri, K. A. Do, X. Wang, F. J. Giles, S. M. O'Brien, H. Saffer, et al., "Circulating CD20 is Detectable in the Plasma of Patients with Chronic Lymphocytic Leukemia and Is of Prognostic Significance," Blood, Vol. 101, No. 7, 2003, pp. 2507-2513. doi:10.1182/blood-2002-06-1639
[16] M. Albitar, K. A. Do, M. M. Johnson, F. J. Giles, I. Jilani, S. O'Brien, et al., "Free Circulating Soluble CD52 as a Tumor Marker in Chronic Lymphocytic Leukemia and Its Implication in Therapy with Anti-CD52 Antibodies," Cancer, Vol. 101, No. 5, 2004, pp. 999-1008. doi:10.1002/cncr.20477

[17] P. Esposito and A. Dal Canton, "CD40/CD40L and Cardiovascular Risk in Patients on Haemodialysis: A Role for Soluble CD40?" Nephrology, Dialysis, Transplantation: Official Publication of the European Dialysis and Transplant Association-European Renal Association, Vol. 26, No. 7, 2011, pp. 2415-2416.

[18] P. Thurmes, T. Call, S. Slager, C. Zent, G. Jenkins, S. Schwager, et al., "Comorbid Conditions and Survival in Unselected, Newly Diagnosed Patients with Chronic Lymphocytic Leukemia," Leukemia \& Lymphoma, Vol. 49, No. 1, 2008, pp. 49-56. doi:10.1080/10428190701724785 